\title{
AIR TRACKING AND MONITORING FOR UNMANNED AIRCRAFT TRAFFIC MANAGEMENT
}

\author{
Iván Campaña, Luca Bergesio, Juan A. Besada, Gonzalo de Miguel
}

\begin{abstract}
The large increase in the sale of drones as well as their use in more and more aspects of daily life has led to the emergence of increasingly voices warning of the need to track and monitoring these drones. The prototype UTM monitoring system to be described in this paper is aimed to address this problem. It is implemented as a microservice to be inserted in a complete UTM SW ecosystem, processing telemetry and other sensors position/velocity information to be able to track, monitor and control the good operation and thus avoid the possible conflicts or accidents that can cause due to a bad use or malfunction of them. This prototype may also allow the analysis of the performance without putting at risk neither people nor material goods since all drone data may be simulated. Also, it has been tested using real data from industrial multirotors.
\end{abstract}

Keywords - UTM, drone, fleet management, unmanned aircraft traffic management system, decision support tools.

\section{Introduction}

According to the European Drones Outlook Study [1], the market of civilian drone applications will be growing extremely fast in the next thirty years. Drone applications can be found in many different articles [2], and some of them are focused on operations to be implemented in urban environments. Drone-based systems are used to enhance functionalities such as surveillance and reconnaissance, monitoring, mapping and photogrammetry, automatic fault detection, inventory tasks, e-commerce and delivery, etc. In this later functionality there are several proposals of the big technology companies due to the huge potential market (e.g. Prime Air service of Amazon [3]). In this scope, air taxies are also a potential future application [4]. Some examples of the use of drones in infrastructure inspection scenarios can be observed in [5], [6] and [7].

The type of flight to be completed is determined by the mission type [8] and there are clear differences for local (Visual Line of Sight VLOS) and remote (Beyond Visual Line of Sight, BVLOS) operation. To obtain cost effective solutions it is necessary that all the applications shown previously have tools to accelerate and partially automate the creation of missions, the calculation of the optimal trajectories and the automatic execution of parts of the mission with the least human intervention. But there are often going to be critical parts in the flights that must be executed by expert pilots due to the higher adaptability of these to mission requirements.

Most of these applications bank on the definition of ad-hoc operations for missions' design, which tend not to consider the flight safety constraints although a safe environment is assumed. But there is a problem in this assumption, and it is that in urban environments there will be a lot of obstacles, areas restricted to flight, areas with height constraints, etc. And there will be an additional problem if the air traffic starts to grow because there will be the necessity to coordinate different operations to avoid drone collisions and to impose physical restrictions to flights as corridors, airways or explicit rules of air in some areas [9]. In the last few years, a collection of concepts and tools have been developed to ensure the safety in the flights. Some examples of these tools are those related to the FAA-NASA UTM (Unmanned Traffic Management [10]) program or to the European U-Space concept from SESAR program [11]. There are not only these tools, but many companies are developing UTM solutions (most of the relevant actors are included in GUTMA association [12]), and the flight regulators (i.e. ICAO, AESA, etc.) are working in opening the sky for these new actors, focused on low level operations and in urban areas. On the part of regulators, it is relevant 
the work of JARUS group [13], which is defining a methodology for risk assessment (called SORA [14]) to automate flight authorization.

UTM is a set of services and functions that working together make the complete functionality. They are implemented using a microservice-based architecture that permits to easily move, replicate and scale the application. Microservices are implemented using the RESTful paradigm, the MQTT protocol for the asynchronous communication, MongoDB for the databases and the JSON format for the messages exchanged among the services. Services are deployed and maintained using docker containers.

In this paper we will describe a particular UTM function developed by Universidad Politécnica de Madrid. This UTM function is the air monitoring, whose main objective is real time flight safety monitoring (encompassing alerting of hazardous situations) based on high performance tracking of unmanned aerial vehicles. We implemented a prototype of this function in a complete microservices based UTM system prototype, to check different concepts of control and management of unmanned aerial traffic and to make a first approximation of what a real tracking and air monitoring service should be like to carry out the management and control of this traffic.

The paper continues with a section devoted to a high-level description of the air monitoring system that we have designed and developed. In the following section we show the initial performance results. In the last section of the paper we present the conclusions and future work.

\section{Air Monitoring System Prototype}

Air monitoring system prototype does all the safety assurance functions related to each individual aircraft: Drone Tracking (UTM tracker), incursion in restricted areas monitoring, deviation from the original flight plan monitoring, obstacle avoidance monitoring, provision to pilots of nearby traffic information and of alerts (including information encompassing manned and unmanned aircraft), among other functions. All this information is showed to the pilots, drone operator managers, state agents (i.e. local police) or UTM operators in a stateof-the-art web/mobile based HMI.
Air monitoring system service is able to track cooperative drones, using cooperative sensor as ADS-B, direct GPS telemetry via mobile, GPS telemetry via through GCS forwarding, etc., providing a single track by drone. This service also supports tracking of non-cooperative drones, by incorporating plots or tracks from non-cooperative sensors as radar, infrared, camera, etc. Also, the system is able to integrate other surveillance systems or UTM system tracks, including ATC/ATM tracks of manned aircraft.

Air monitoring system is being continuously developed and updated through versions to allow for the progressive implementation of changes.

This system offers the following basic functions:

- Processing of all drones in flight through telemetry that it is sent to the air monitoring system.

- Processing of ADS-B data from equipped drones.

- Processing of non-cooperative sensors information as radar, infrared and camera for the functions of aircraft monitoring and identification and for maintaining air traffic separation.

- Processing of other surveillance systems or UTM system tracks, including ATC/ATM tracks and presentation. The idea is to be able to work as a federation of monitoring services from different vendors, being able to adapt to different UTM architectures.

- Presentation of drones in flight related to pilot, drone operator, state agents, or UTM operator. Each user can only see the drones allowed to its role and ownership, and the ones that conflict with theirs, so that all privacy constraints are respected.

- Processing of all flight plan information and correlation with tracks, based on both telemetry or other sensors (ADS-B, ...) identifications and on positional information.

- Alerts to users of aircraft deviations from the planned route after comparing the telemetry received and the flight plan created and authorized. 
- Alerts to users of incursions in restricted areas or areas with a set of rules that must be satisfied.

- Alerts to users of obstacles presence comparing telemetry sent by the drone and the AGL height obtained making use of a high resolution and accuracy ground service.

- Presentation of weather information to assist with navigation in adverse conditions.

- Presentation of the necessary aeronautical information to the pilot.

- Distributed monitoring, configuration, recording and technical exploitation of the entire system.

Air monitoring system is composed by six modules, each of one have a function, but together the system offers all the functions that have been exposed before. A scheme of the system can be observed in Figure 1. Below are the six modules that make up the system and in the following subsections are explained these in detail.

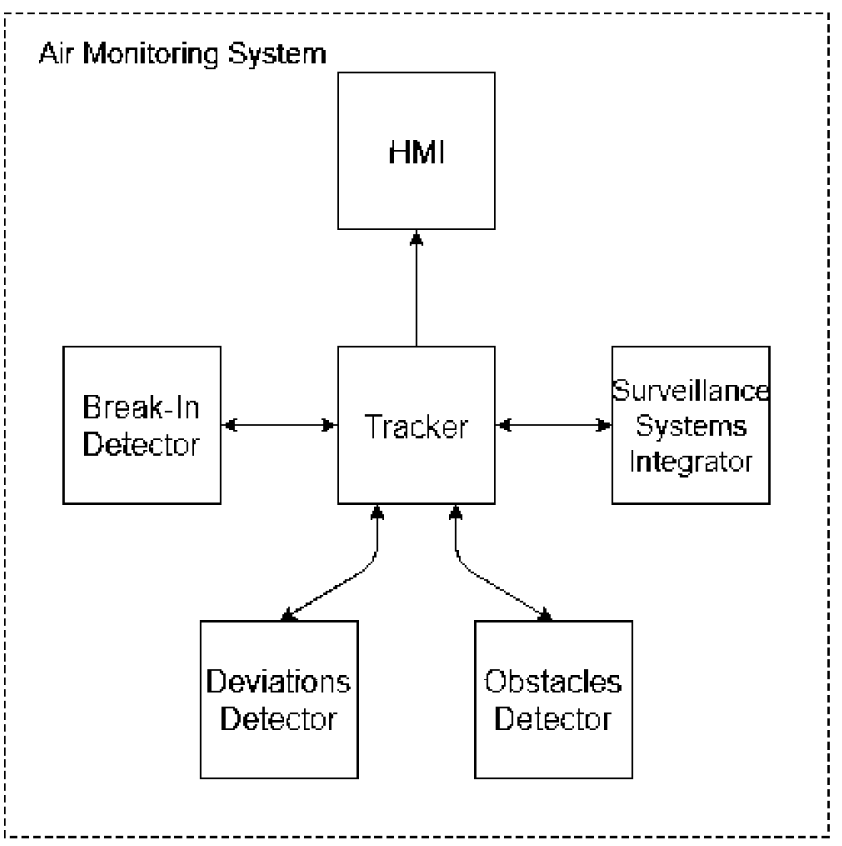

Figure 1. Scheme of the System

The six modules are:

- Tracker

- Break-In Detector
- Deviations Detector

- Obstacles Detector

- Surveillance Systems Integrator

- HMI

\section{Tracker}

To be able to detect and track all the drones simultaneously, it has been implemented two Bayesian filters [15] for each track making use of the flight plan information to improve tracking. One is a horizontal filter where it is predicted and filtered the $\mathrm{x}$ and $\mathrm{y}$ positions and the $\mathrm{x}$ and $\mathrm{y}$ speed. The other one is a vertical filter where the $\mathrm{z}$ position and the $\mathrm{z}$ speed are predicted and filtered.

The structure implemented to carry out the tracker function can be seen in Figure 2 and the operation of this air monitoring system service is explained in detail below.

Each drone sends the telemetry that measures every second (with a certain temporary tolerance) and this telemetry is received.

On the one hand, telemetry correlate with available detailed flight plans formed of a collection of flight segments. In this way, the trajectory that the drone will follow can be predicted, and the system may also predict the segment of the flight plan the drone is flying at the current time.

Once the flight segment is determined a flightsegment adapted Bayesian tracker is reached. In this tracker, in addition to having the telemetry information that the drone is sending, the information associated with the flight segment of the correlated flight plan is available. In this way, a more precise filtering and estimation can be done since more flight context information is available (flight segment orientation, desired baseline).

On the other hand, telemetry is also sent to a robust Bayesian tracker in which it uses only the information that the drone is sending to predict and filter the position and speed of the drone. This Bayesian tracker is capable of estimating accurately drone position even if the drone is not following a flight plan, or when the flight plan specification is not composed of detailed flight segments, but only of flight areas. 


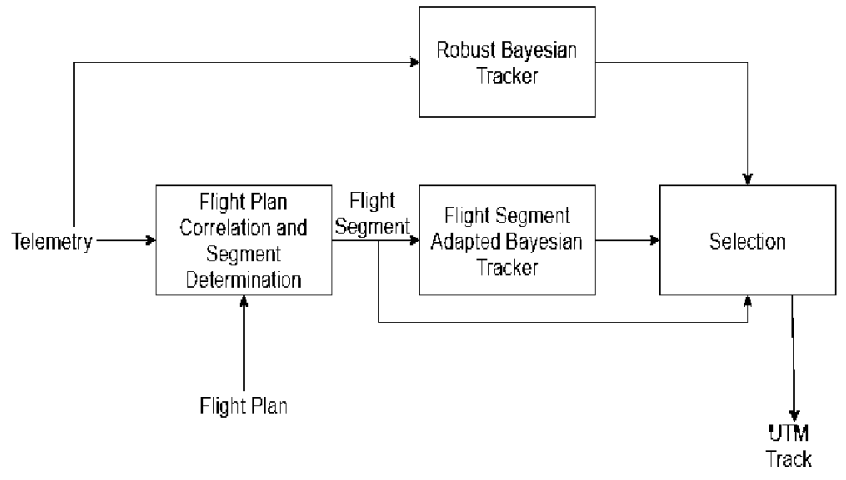

Figure 2. Tracker

Once the positions and speeds of the drone have been calculated by both trackers, the track selector chooses the output to be provided. If it does not have a correlated flight plan with the telemetry that the drone has sent, the track that leaves the robust Bayesian tracker is selected as the output UTM track. If, on the other hand, the correlated flight plan is available, the selector has the track obtained with the flight segment adapted Bayesian tracker as the output UTM track.

\section{Break-In Detector}

The regulation of drones is different for different scenarios, but in general it is clear about the conditions under which a drone can fly. Next, we are focusing in the Spanish regulation. Basic rules state flights must be performed at less than 120 meters high, never over populated areas or special buildings and always maintaining eye contact. But there are geographical areas where a drone cannot be flown.

Flying drone is not allowed in all airspace. There are areas delimited by which it can not be flown. These zones are stipulated by the relevant organizations, as the government of a country, the government of a city, a company, an individual... The Spanish regulation [16] is ruled by the Ministry of Public Works, and sometimes by the Ministry of Defense. Even if you have the approval of the mayor to fly over a town hall or your neighbor to fly over your house, the final permit depends only on the air safety agency, or AESA.

The Spanish airspace is within the European FIR and, in turn, it is divided into three smaller regions:
Madrid, Barcelona and the Canary Islands. These regions are also divided into several volumes, which are stacked on top of each other. According to current Spanish regulations, RPAS are prohibited from entering areas of controlled airspace and limited airspace zones.

Under common names internationally, there are several types of controlled airspaces. These can be used by aircraft under certain circumstances

- CTA or control area. It serves to protect aircraft from the moment they take off until they enter a route or an airway. CTAs are formed by a volume of controlled airspace in the vicinity of airports.

- TMA or terminal area of maneuvers. It is the same as the CTA, but for airports with more traffic.

- CTR or control area. One of the best known among drone pilots, it forms a bubble around each airport to protect incoming and outgoing traffic.

- ATZ or aerodrome transit area. It covers the movement of aircraft in a volume of about eight kilometers in radius and less than one kilometer in height around airports.

- AWY or airway of air route. They are defined corridors that connect two geographical points to a certain altitude.

In addition to controlled areas, drones cannot fly over limited airspace, as do most aircraft. According to Spanish legislation, four types are recognized:

- Prohibited. In the aeronautical charts it appears marked as LEP. To fly over it, express permission from the Ministry of Defense is required. LEP are, for example, nuclear power plants or real estate properties.

- Dangerous. These are identified as LED. Some aircraft can fly over these areas as long as they make sure that no dangerous activity is taking place. They are, for example, military practice zones.

- Restricted. These areas are indicated in the aeronautical charts as LER and can only be flown over by state aircraft in case of emergency. As examples are the corridor 
of Algeciras or national parks such as Picos de Europa.

- Areas with sensitive fauna. These are protected areas that are identified with the letter $\mathrm{F}$

It is for all this that it is necessary to have a break-in detector in restricted areas to let the users (and especially state agents) know that this drone is committing an infraction that must be checked, because it may be the case that this drone has the necessary permissions to make that incursion.

For this purpose, the location information of all these areas in the Spanish territory is available in first approximation. Once they have it, it is verified that the position of the drones, as provided by the tracking system, is not inside any of these areas. In the case the drone is not estimated to be inside the area, this detector does not send any alert, but it indicates the minimum distance to any one of these restricted/protected areas. If, on the contrary, this module detects that the drone is inside one of these restricted or forbidden areas, the detector sends an alert to all involved users saving that it is entering an area where it can not be flown and that the appropriate measures will be taken in the case the drone does not have the necessary permits, so leave that area as soon as possible.

\section{Deviations Detector}

A good drone flight plans tracker must take into account the original flight plan that is intended to fly. It is not enough to know at all times the position of a drone, but it is necessary to know if the established flight plan is followed, because if it is diverting more than it is possible, it can have different types of problems and be a threat not only for the rest of drones in the airspace, if not also for people. But not only in terms of position, but also with respect to time, because a drone going slower or faster than the established, or departing at not authorized times can affect the operation of other drones and end up causing accidents.

For these reasons, there must be a comparison between the generated flight plan and the monitored target state according to the tracking system. At each time it is received the position of the drone, it is checked if the drone is in the position it should be at the planned time interval, always with a certain margin of error, to take into account both flight operation uncertainty and tracking errors.

The flight plans can be a time-ordered combination of two different specifications, described more in depth in [17]:

- Linear segments to be followed by the aircraft, with associated height and time constraints

- Volumes, where the flight must be confined for a given time interval (duration), also with associated height constraints.

For the first kind of specifications, lateral, height and time deviations are checked. In the case where the deviation that occurs in the lateral component is very large, an alert is sent to indicate that the drone is flying outside of its established flight plan. If the deviation is small, only the deviated meters are indicated but no alert is sent. The same happens with the deviation in height and the deviation in time, being these less restrictive since there is a restriction of height and minimum time and a restriction of height and maximum time.

For volume-related flight plan specifications, it is checked the track is inside the volume for the intended time interval.

Therefore, if any of these restrictions is breached, the detector sends the alarm directly saying the reason why it is launched.

\section{Obstacles Detector}

Apart from knowing always the height at which the drone is flying and its deviation from the programmed height, it is important to know the terrain on which the drone is flying to avoid collisions with the ground or obstacles that appear along the way.

When planning a flight plan, it is done knowing the height of the ground to fly at a distance from it and thus avoid collisions. In addition, a guard margin is added to take into account both flight technical error and altimeter errors. 
In any case, the system compares the height of the drone it is flying with the height of the terrain in the position where the drone is, issuing an alert if the drone is to near the ground while in the middle of the flight. At that time, it is necessary to analyze if what is happening is that the drone is descending slowly because it is going to land not issuing an alert in this case.

\section{Surveillance Systems Integrator}

Until now, everything explained in terms of calculating the alarms in the respective detectors is associated with the positions of the drones that they send by telemetry at each moment. But there may be drones not send telemetry must also be tracked and monitored by this system (if drone positional data is obtained from other sensors).

To prevent this from happening, it is necessary to incorporate other surveillance systems in the UTM system that send the necessary information to track and monitor all the drones. Other surveillance systems that can be incorporated into the original UTM system are:

- Radars. There are radars that can detect small, slow and low-flying targets, while others can detect larger targets and move with greater speed [18]. For these reasons, radars are suitable for tracking and monitoring drones.

- Cameras. Another way in which the detection of UAVs is performed is the one developed in [19] and [20]. These systems consist of a set of tracking architectures composed of cameras that calculate the destination trajectories of the aircraft projected in the camera. These surveillance systems are often used in the nearest of an airport.

- Acoustic sensors. An array of acoustic sensors can be used to detect and estimate the direction of arrival of sounds from sources such as UAVs. An example of implementation of this type of detection of UAVs is explained in [21].

- Other methods as Wi-Fi detection can operate as a surveillance system. This procedure is explained in [22] and [23].
All these sensor systems will send to air monitoring system prototype the information that they capture for each drone that they are capable of detecting. Once in the system, it will be necessary to convert this type of data to the one used internally in order to operate properly and thus track and monitor those drones that do not send telemetry.

But not only are these other surveillance systems integrated in this module here explained. The emergence of drones led to the need for the creation of a control service and management of drone air traffic, but until then there was already a system of control and management of air traffic for large aircraft (ATM/ATC). The tracking information from the ATM/ATC, that is in Asterix 62 format [24], must be translated to the data used internally in UTM service and in this way, the air monitoring system is able to track and monitoring the aircrafts, not only the drones. On the other hand, the data from UTM service must be translate to the form employed in the ATM/ATC service so the drones can be tracked and monitored in that service.

Figure 3 shows the process that is carried out in the Surveillance System Integrator. On the one hand, it is received the telemetry that is entered in the tracked and it is get the UTM tracks, on the other hand it is received the tracks of the sensors (one for each sensor that sends information) and an alignment of the data is made according to the data employee in the system, and finally ATC tracks are received in Asterix 62 format and converted into UTM tracks. Once all these tracks have a data fusion and they are provided as output in the air monitoring system HMI. Also, the system has a gateway to make its data available to ATC systems using Asterix 62 format.

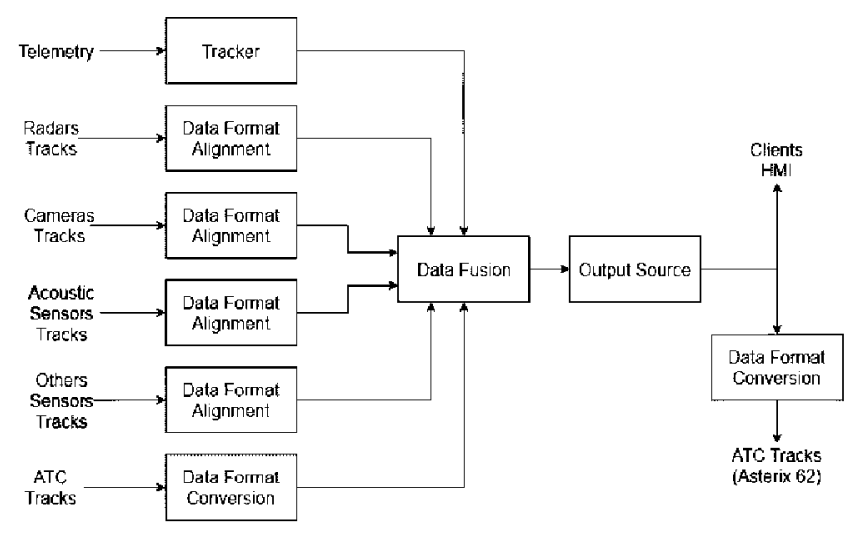

Figure 3. Surveillance Systems Integrator 


\section{HMI}

Everything explained above is related to the calculations and the internal logic that the prototype needs to work correctly, but without a graphical interface where it can be seen the results, the system is nothing. Human to Machine Interface (HMI) makes it easier for human to manage and interpret measurements and readings from instruments. This leads to an increase in productivity because less time is required to ensure that the systems or the instruments are working correctly. Also leads to a safer working environment. The development of airground traffic management systems, and now air tracking and monitoring systems for unmanned aircraft traffic management, changes human roles and responsibilities in the airspace system. These changes create new challenges for the development of effective Human to Machine Interfaces (HMIs) for air traffic control. These HMIs must be developed to allow users to visualize air traffic information and quickly identify airspace areas where user intervention is required without creating information overload or excessive workload.

It is for these reasons that it is needed a graphical interface where it can be tracked and monitored all drones of a certain area, as well as taken the relevant alarms that the different detectors throw, although the system behind it is doing so in all drones that are in the system and even though all the information about positions, follow-ups and alarms is presented and sent in the previous modules.

In this system there are different types of users. Depending on the user, it has more restrictive or less restrictive vision permits, so they can see more or less drones.

To perform this type of filtering based on the user that is, the module has to connect with other services that are not the subject of this paper, so they are not explained in detail. From these services you get the users and drones that you have, and you are allowed and compare with those that arrive in the request to this module on the other hand, and those that concur are those that are shown and followed by this module.

An example of the graphical interface that is used for this air monitoring system prototype is the one that can be seen in Figure 4.

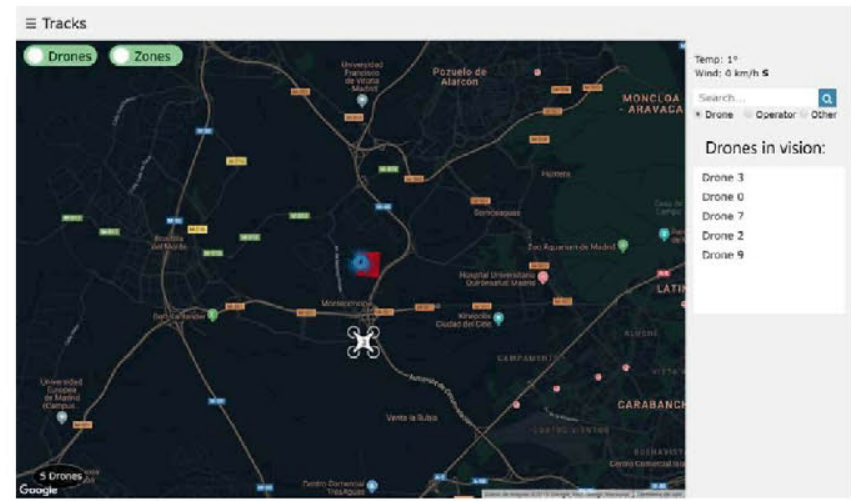

Figure 4. Example Image of HMI

\section{Initial Results}

The results presented next are not definitive, as this is still an on-going process. Currently, the basic tracker, detector and monitoring chain is completed, but we still do not have higher level decision support in the systems implemented as a lot of other surveillance system integrated.

All the results shown are with the drones simulated, although the system has also been used with real DJI drones, using an in-house developed GCS. In Figure 4 an output of the air monitoring system with a drone monitored is depicted. In this picture it may be seen 5 different drones detected as it is said in the ellipse on the bottom left corner. One of these drones (the one which has the number 3 ) is seen with a drone draw because it doesn't present any problem. On the contrary, the other four drones can present problems because they are grouped and represented as a blue circle, so it is necessary to do zoom-in to see in more details. If the information of the tracked drone needs to be showed it must click on the drone and it is showed as it can be seen in Figure 5. These figures are only images taken from the air monitoring service that is working in real time, so in this service the drones are tracked and monitored satisfactorily and at no time false alarms appear from the detectors that would damage the correct performance. 


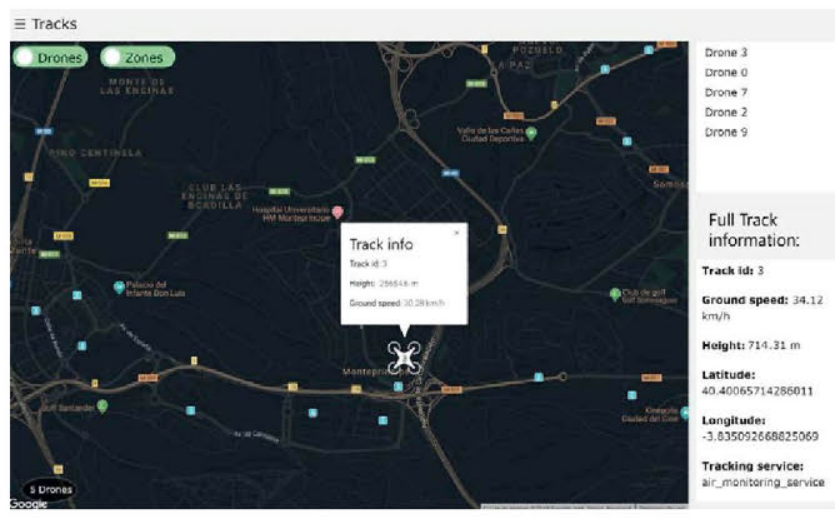

Figure 5. Information About a Drone

Below are some results of the detectors demonstrating their proper functioning. In Figure 6 it can be seen how three drones are entering a restricted or prohibited area and change color warning of their failure to comply with the rules. Therefore, the Break-In detector is fulfilling its purpose.

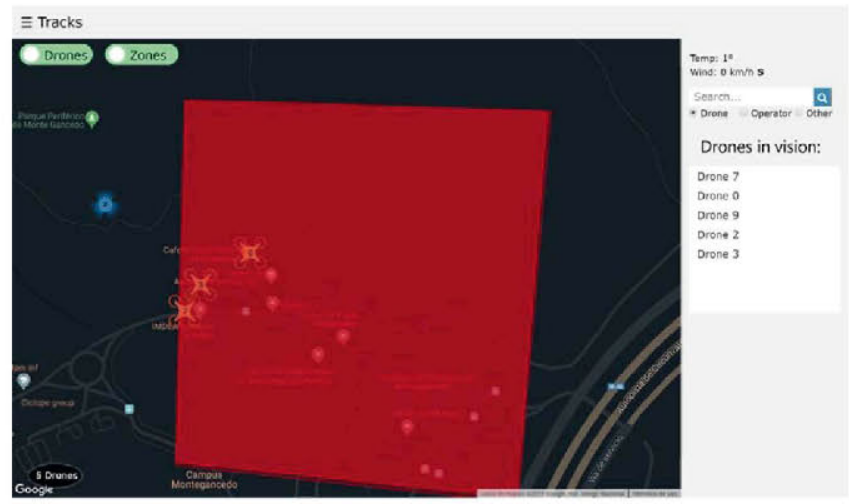

Figure 6. Example of Break-In Detector

\section{Conclusions}

The paper describes an air monitoring system prototype. The prototype functionalities in detail, and also their interrelations, the implementations constraints and the complete HW/SW deployment to model a typical drone tracker and monitor scenario are described.

The potential benefits of the prototype elaborated to perform are:

- It may be used to research on user interfaces for drones monitoring and tracking operations.

- It also may be used to design decision support tools and infer their results in a real scenario that can be given at the time it is flying a drone. It may therefore serve as a design/training tool to gain expertise in the creation of a real and bigger UTM service with an air monitoring service, helping the developed solutions allow guaranteeing an efficient service (hopefully reducing reaction times, increasing safety, reducing the stress of the controller and its workload, etc.)

- It may be used as a showcase to demonstrate those new technologies.

- Finally, it may be used for educational purposes for ATM, UTM, drones and technology applications systems.

\section{References}

[1] SESAR Undertaking, "European Drones Outlook Study-Unlocking the Value for Europe", SESAR, Brussels, 2016.

[2] M. \&. A. A. Hassanalian, "Classifications, Applications, and Design Challenges of Drones: A Review", Progress in Aerospace Sciences, 2017.

[3] Amazon. Amazon Prime Air, 2016. Available online at https://www.amazon.com/Amazon-PrimeAir/b?node $=8037720011$.

[4] Stimpson, A., Cummings, M., Nneji, V. C., \& Goodrich, K. H. (2017). "Exploring Concepts of Operations for On-Demand Passenger Air Transportation".

[5] Ariff, O. K. (2017). "Introduction to Unmanned Aircraft Systems", Second edition DM Marshall et al CRC Press. The Aeronautical Journal, 121(I244), 1592-1595.

[6] Besada, J. A., Bergesio, L., Campaña, I., Vaquero-Melchor, D., López-Araquistain, J., Bernardos, A. M., \& Casar, J. R. (2018). "Drone Mission Definition and Implementation for Automated Infrastructure Inspection Using Airborne Sensors". Sensors, 18(4), 1170.

[7] Barkham, R., Bokhari, S., \& Saiz, A. (2018) "Urban Big Data: City Management and Real Estate Markets". GovLab Digest: New York, NY, USA.

[8] González-Jorge, H., Martínez-Sánchez, J., \& Bueno, M. (2017). "Unmanned aerial systems for civil applications: A review". Drones, 1(1), 2. 
[9] Airbus. Premiering a future blueprint for our sky, 2018. Available online at: https://www.airbus.com/innovation/Premiering-afuture-blueprint-for-our-sky.html.

[10] NASA. FAA-NASA UTM, 2015. Available online at: https://utm .arc.nasa.gov/index.shtml.

[11] SESAR, 2014. Available online at: https://ec.europa.eu/transport/modes/air/sesar_en.

[12] Global UTM Association. Available online at: https://gutma.org/.

[13] JARUS: Joint Authorities for Rulemaking on Unmanned Systems. Available online at: http://jarusrpas.org/.

[14] SORA: Specific Operations Risk Assessment (SORA), JARUS, 2017. Available online at: http://jarus-rpas.org/content/jar-doc-06-sora-package.

[15] López-Araquistain, J., Jarama, Á. J., Besada, J. A., de Miguel, G., \& Casar, J. R. (2019). A new approach to map-assisted Bayesian tracking filtering. Information Fusion, 45, 79-95.

[16] BOE: Boletín Oficial del Estado. Available online https://www.boe.es/boe/dias/2017/12/29/pdfs/BOEA-2017-15721.pdf

[17] Besada, J., Campaña, I., Bergesio, L., Bernardos, A. M., \& de Miguel, G. (2019). Drone Flight Planning for Safe Urban Operations: UTM Requirements and Tools. UNAGI 2019: Workshop on Unmanned aerial vehicle Applications in the Smart City: from Guidance technology to enhanced system Interaction, Kyoto, 11-15 March 2019.

[18] Krátký, M., \& Fuxa, L. (2015). Mini UAVs Detection by Radar. IEEE, Military Technologies (ICMT), 2015 International Conference on.

[19] Besada, J., Portillo, J., García, J. M., Varona, A., \& González, G. (2005). Image-Based Automatic Surveillance for Airport Surface.

[20] Besada, J., Portillo, J., García, J., Molina, J., Varona, A., \& González, G. (2005). Airport Surface Surveillance for Airport Surface.
[21] Benyamin, M., \& Goldman, G. H. (2014). Acoustic Detection and Tracking of a Class I UAS with a Small Tetrahedral Microphone Array. ARL.

[22] Peacock, M., \& Johnstone, M. (2014). Detection and Control of Small Civilian UAVs. School of Computer and Security Science, Edith Cowan University.

[23] Peacock, M., \& Johnstone, M. N. (2013). Towards detection and control of civilian unmanned aerial vehicles. School of Computer and Security Science, Edith Cowan University Security Research Institute.

[24] Eurocontrol Standard Document for Surveillance Data Exchange Part 9: Category 062 Transmission of System Track Data. Available online at:

https://www.eurocontrol.int/sites/default/files/content /documents/nm/asterix/archives/asterix-cat062system-track-data-part9-v1.12-092010.pdf

\section{Acknowledgements}

This work was supported in part by Universidad Politécnica de Madrid Project "Tecnologías Avanzadas para la Monitorización y Gestión Remota del Tráfico Aéreo de Vehículos Pilotados y no Pilotados" (RP1509550C02), by Fundacion para el Desarrollo de las Telecomunicaciones (FUNDETEL) Project "Tecnologías y Sistemas de Control y Gestión de Tráfico Aéreo" (GPD.SJC.001) and by the Spanish Ministry of Economy and Competitiveness under Grant TEC2017-88048-C2-1-R.

2019 Integrated Communications Navigation and Surveillance (ICNS) Conference

April 9-11, 2019 